\title{
ESTUDO DA DISTRIBUIÇÃO DA EVAPOTRANSPIRAÇÃO DE REFERÊNCIA VISANDO O DIMENSIONAMENTO DE SISTEMAS DE IRRIGAÇÃO.
}

\author{
João Carlos Cury.Saad \\ Guilherme Augusto Biscaro \\ Osmar Delmanto Jr. \\ Dept ${ }^{\circ}$ de. Eng. Rural, FCA/UNESP, CP. 237, CEP 18603-970 - Botucatu, SP. \\ José Antonio Frizzone \\ Depto. Eng. Rural, ESALQ/USP, CP. 09, CEP 13419-900 - Piracicaba, SP.
}

\section{RESUMO}

Visando caracterizar a distribuição de freqüência da evapotranspiração de referência na região de Piracicaba, SP, e avaliar as práticas usuais de estimativa da necessidade de água para fins de dimensionamento de sistemas de irrigação, foram utilizados 30 anos de dados de evapotranspiração do mês de setembro, que foi dividido em períodos de 5, 10, 15 e 30 dias. As distribuições Beta e Normal foram aplicadas aos dados e ambas mostraram-se aptas para representá-los. Para valores de probabilidade de ocorrência iguais ou superiores a $60 \%$, verificou-se o aumento do valor da evapotranspiração de referência com a diminuição do período. Adotando como parâmetros o período de máxima exigência hídrica de 2 a 3 semanas e a evapotranspiração de referência ao nível de $75 \%$ de probabilidade, verificou-se que o uso do valor médio mensal da evapotranspiração no dimensionamento de sistemas de irrigação conduz ao subdimensionamento, enquanto a adoção do máximo valor diário da evapotranspiração acarreta o superdimensionamento.

UNITERMOS: Evapotranspiração de referência, distribuição de freqüência, dimensionamento de sistemas de irrigação.

\section{SAAD, J. C. C., FRIZZONE, J. A., BISCARO, G. A., DELMANTO JR., O. STUDY OF THE REFERENCE CROP EVAPOTRANSPIRATION DISTRIBUTION RELATED TO IRRIGATION SYSTEM DESIGN.}

\section{ABSTRACT}

This paper has studied 30 year reference crop evapotranspiration data distribution aiming to improve irrigation system design. Evapotranspiration was analyzed using 5,10, 15 and 30 day - time periods over the month of September, for the region of Piracicaba city, São Paulo state. Normal and Beta distribution have been applied to data and through Kolmogorov-Smirnov test their adequacy to represent reference crop evapotranspiration data has been verified. An increase of reference crop evapotranspiration value has been obtained for occurrence probability values equal or higher than $60 \%$ 
when the period of time has been shortened. Considering 2 to 3 weeks the water requirement peak period and $75 \%$ probability the reference crop evapotranspiration, it could be observed that using monthly mean value of evapotranspiration to get irrigation system design leads to its underestimation whereas maximum daily value of evapotranspiration leads to its overestimation.

KEYWORDS: frequency distribution, reference crop evapotranspiration, irrigation systems design.

\section{INTRODUÇÃO}

A evapotranspiração de referência é uma variável fundamental para a estimativa da exigência em água das culturas, a qual irá determinar o dimensionamento dos sistemas de bombeamento, de adução, de distribuição e de aplicação de água, característicos de cada método de irrigação.

A diversidade de métodos de estimativa da evapotranspiração de referência e a grande variabilidade de valores assumidos pelos parâmetros meteorológicos durante o período de máxima exigência hídrica das culturas irrigadas, acarretam considerável dispersão dos valores calculados da evapotranspiração, sugerindo uma análise da distribuição de freqüência dos valores estimados, para fins de dimensionamento de sistemas de irrigação (Saad \& Scaloppi, 1988).

Pruitt et al. (1972), trabalhando com dados de lisímetro instalado em Davis, EUA, obtiveram a distribuição de freqüência da evapotranspiração diária para todos os meses do ano, em períodos de $1,3,5,7,15$ e 30 dias, válida para a região central da Califórnia, EUA. Trabalho semelhante foi realizado por Nixon et al. (1972) para a região costeira da Califórnia e por Wright \& Jensen (1972), para a região sul de Idaho, EUA.

A análise da distribuição de freqüência dos dados mensais de evaporação do tanque IA-58, para Ribeirão Preto, SP, foi conduzido por Arruda \& Barroso (1984). Aplicando a distribuição Normal, os autores concluíram que os valores mensais de evaporação correspondentes ao nível de $75 \%$ de probabilidade foram significa-

Este trabalho tem por objetivos identificar a distribuição de probabilidade teórica capaz de representar a evapotranspiração de referência para o mês de setembro na região de Piracicaba, SP, e avaliar o efeito tanto do uso do valor médio mensal como do tivamente superiores aos valores médios mensais registrados no período.

Saad \& Scaloppi (1988) aplicaram o método de Penman-Monteith a dados meteorológicos de Piracicaba, SP, e realizaram estudos sobre a distribuição de freqüência das estimativas de evapotranspiração, utilizando a distribuição Normal. Os resultados obtidos permitem que se proceda à seleção de valores representativos da evapotranspiração, em períodos consecutivos de 1 a 30 dias, e níveis de probabilidade variáveis entre 2 e $98 \%$.

Yao (1969) verificou que a distribuição de freqüência de um índice " $R$ " relacionado com a evapotranspiração de referência ajustou-se satisfatoriamente à distribuição Beta, para períodos de quinze dias e mensais.

Os sistemas de irrigação são, de maneira geral, dimensionados com base no valor médio mensal da evapotranspiração ou em função do valor máximo diário durante o período de maior exigência hídrica da cultura. Estes dois critérios são bastante distintos quanto à duração do período de pico em termos de exigência hídrica e, também, quanto à probabilidade de ocorrência destes valores de evapotranspiração, definida pela distribuição de freqüência dos dados. Além disto, eles diferem de citações de literatura, as quais indicam que o período de máxima exigência hídrica das culturas irrigadas é de, geralmente, duas a três semanas (Jensen, 1974) e que, na maioria das regiões irrigadas, utiliza-se a evapotranspiração de referência ao nível de $75 \%$ de probabilidade de ocorrência, para fins de dimensionamento de sistemas de irrigação (Doorenbos \& Pruitt, 1984).

\subsection{Distribuição Beta}

A distribuição acumulada Beta incompleta $\mathrm{F}(\mathrm{x}$ ') fornece a probabilidade de que a evapotranspiração de referência não 
valor máximo diário da evapotranspiração durante o pico de exigência hídrica, em relação ao critério recomendado que estabelece período de maior demanda entre duas e três semanas e adoção da evapotranspiração ao nível de $75 \%$ de probabilidade de ocorrência.

\section{MATERIAL E MÉTODOS}

A evapotranspiração de referência foi estimada utilizando dados climáticos de um período de 30 anos (de 1950 a 1968, 1977, 1979, 1981 a 1989), referentes ao mês de setembro, coletados no Posto Agrometeorológico do Departamento de Física e Meteorologia da ESALQ-USP, localizado em Piracicaba, SP. O mês de setembro foi dividido em períodos de 5,10,15 e 30 dias e a sua escolha para exemplificar este estudo deve-se ao fato de que, na maioria das vezes, ele corresponde ao período onde se concentra a máxima exigência hídrica das culturas irrigadas plantadas em abril ou maio. As estimativas de evapotranspiração foram utilizadas na forma de valor médio, expresso em mm/dia.

\subsection{Estimativa da evapotranspiração de referência}

O método selecionado para estimativa da evapotranspiração de referência foi o de Penman, tendo sido utilizado com as necessárias adaptações para aplicação na região de Piracicaba, SP, detalhadamente descritas em Saad (1990). A cultura de referência adotada foi a grama forquilha (Paspalum notatum, L.) por ser a mais utilizada para este fim, nas condições brasileiras.

Para analisar a distribuição de freqüência das estimativas da evapotranspiração foram avaliadas as distribuições Beta e Normal. exceda, em um dado período, um valor x', sendo representada pela equação (Falls, 1973):

$F\left(x^{\prime}\right)=\frac{\Gamma(\mathrm{p}+\mathrm{q})}{\Gamma(\mathrm{p}) \Gamma(\mathrm{q})} \cdot \int_{0}^{x^{\prime}} x^{\prime}{ }^{p-1} \cdot\left(1-\mathrm{x}^{\prime}\right)^{\mathrm{q}-1} \cdot d x^{\prime}$

$$
\mathrm{x}^{\prime}=\frac{\mathrm{x}-\mathrm{a}}{\mathrm{b}-\mathrm{a}}
$$

onde,

x'- variável evapotranspiração de referência transformada pela equação 2 , de tal forma que $0 \leq \mathrm{x}^{\prime} \leq 1$;

x- valor qualquer da variável em estudo (evapotranspiração), compreendido no intervalo $(a, b)$;

a- menor valor da série de dados de evapotranspiração;

b- maior valor da série de dados;

p e q- parâmetros da distribuição Beta, sendo $p$ $>0$ e q $>0$

$\Gamma$ - símbolo da função Gama.

A equação 1 fornece a probabilidade de ocorrência de uma evapotranspiração de referência menor ou igual a um valor x'. Para se obter o inverso, ou seja, partindo-se de um valor de probabilidade encontrar o valor correspondente de evapotranspiração de referência, pode-se utilizar a técnica de interpolação.

Os parâmetros p e q da distribuição Beta podem ser estimados utilizando-se o método dos Momentos (Pearson, 1934):

$\mathrm{p}=\frac{\mu_{1} \cdot\left(\mu_{1}-\mu_{2}\right)}{\mu_{2}-\mu_{1}^{2}}$ 


$$
\begin{aligned}
& q=\frac{\left(1-\mu_{1}\right) \cdot\left(\mu_{1}-\mu_{2}\right)}{\mu_{2}-\mu_{1}^{2}} \\
& \mu_{1}=\sum_{i=1}^{j} \frac{x_{i}^{\prime}}{j} \\
& \mu_{2}=\sum_{i=1}^{j} \frac{x_{i}^{\prime 2}}{j}
\end{aligned}
$$

onde,

$\mu_{1}$ - momento de ordem 1 para a variável x';

$\mu_{2}$ - momento de ordem 2 para a variável x';

$\mathrm{X}^{\prime}{ }_{\mathrm{i}}$ - evapotranspiração transformada pela equação 2 ;

j - número total de dados que compõem a série analisada de valores de evapotranspiração (neste estudo, $\mathrm{j}=30$ ).

\subsection{Distribuição Normal}

A função densidade de probabilidade da distribuição Normal e os procedimentos para cálculo da distribuição acumulada podem ser encontrados em Spiegel (1985).

\subsection{Teste de Kolmogorov-Smirnov}

Para verificar o ajuste dos dados precipitação pluvial às distribuições de probabilidade teóricas, aplicou-se o teste de Kolmogorov-Smirnov. Segundo Campos (1983), este teste verifica a adaptação de uma específica e bem conhecida distribuição a dados provenientes de uma distribuição desconhecida.

\section{RESULTADOS E DISCUSSÃO}

As estimativas da evapotranspiração de referência relativas aos períodos de $5,10,15$ e 30 dias do mês de setembro (Tabela 1)foram subme- tidas ao teste de Kolmogorov-Smirnov para verificar se poderiam ser representadas pelas distribuições Beta e/ou Normal. A hipótese Ho de cada uma destas duas distribuições foi aceita para todos os períodos analisados, ao nível de significância de 5\%. Portanto, a distribuição Beta e a distribuição Normal estão estatisticamente aptas para representar os dados de evapotranspiração de referência do mês de setembro, em Piracicaba, SP.

Aplicando-se a distribuição Beta aos dados de evapotranspiração de referência do mês de setembro, foram obtidos os diferentes níveis de probabilidade de ocorrência, para os vários períodos analisados (Tabela 2). Estes níveis indicam a probabilidade de ocorrência de valores de evapotranspiração iguais ou inferiores aos calculados pela distribuição. Por exemplo, existe $75 \%$ de probabilidade de que o valor da evapotranspiração de referência para a primeira quinzena do mês de setembro não ultrapasse 4,5 $\mathrm{mm} /$ dia. Outra forma de interpretação deste valor consiste em supor que, em três de cada quatro anos, a evapotranspiração será igual ou menor que 4,5 $\mathrm{mm} /$ dia. Em condições de irrigação tipicamente suplementar, como se verifica na região centro-sul do Brasil e, consequentemente, na região de Piracicaba, $\mathrm{SP}$, dificilmente a economia dos projetos de irrigação justifica a escolha de níveis de probabilidade de ocorrência superiores a $90 \%$. Doorenbos \& Pruitt (1984) revelam que na maioria das regiões irrigadas esses níveis variam entre 75 e $80 \%$. Em termos de Brasil, o que se verifica, na prática, é a utilização do valor médio mensal da evapotranspiração ou a adoção do valor máximo diário do período de maior exigência hídrica da cultura irrigada.

Para comparar o uso do valor médio mensal em relação ao valor recomendado, correspondente ao nível de $75 \%$ de probabilidade, montou-se a Tabela 3.

Observa-se que os valores médios dos períodos eqüivalem ao nível de probabilidade de $50 \%$, o que é coerente pois os dados ajustaram-se à distribuição Normal, na qual a linha vertical de simetria passa pela média aritmética, o que confere a este valor $50 \%$ de probabilidade de ocorrência.

Verifica-se, ainda, que a faixa de varia- 
ção entre o valor da evapotranspiração de referência a $75 \%$ de probabilidade e o valor médio, para os diferentes períodos, foi de 7,1 a $16,7 \%$, sendo que os maiores desvios ocorreram nos menores períodos.

Assumindo-se que o procedimento mais adequado é aquele que adota a evapotranspiração em torno de $75 \%$ de probabilidade, verifica-se que existe um subdimensionamento ao se utilizar o valor médio, embora de pequena magnitude.

Tabela 1. Valores da evapotranspiração de referência (em $\mathrm{mm} / \mathrm{d})$ para os períodos de 5, 10, 15 e 30 dias do mês de setembro, durante um intervalo de 30 anos (Piracicaba, SP).

\begin{tabular}{cccccccccccc}
\hline $\mathrm{S}(5$ & $\mathrm{S}(5)$ & $\mathrm{S}(5)$ & $\mathrm{S}(5$ & $\mathrm{S}(5$ & $\mathrm{S}(5)$ & $\mathrm{S}(10$ & $\mathrm{S}(10$ & $\mathrm{S}(10$ & $\mathrm{S}(15$ & $\mathrm{S}(1$ & $\mathrm{S}(30$ \\
\hline 5,2 & 5,8 & 5,5 & 5,2 & 5,4 & 5,5 & 5,4 & 5,4 & 5,4 & 4,9 & 4,9 & 4,9 \\
5,1 & 5,3 & 5,3 & 5,2 & 5,3 & 5,4 & 5,1 & 5,0 & 4,6 & 4,9 & 4,8 & 4,7 \\
5,0 & 5,2 & 5,0 & 5,2 & 4,9 & 5,1 & 4,9 & 5,0 & 4,6 & 4,7 & 4,7 & 4,5 \\
4,7 & 5,0 & 4,9 & 5,1 & 4,9 & 5,1 & 4,6 & 4,9 & 4,5 & 4,7 & 4,6 & 4,5 \\
4,6 & 4,9 & 4,9 & 4,9 & 4,9 & 5,0 & 4,5 & 4,8 & 4,5 & 4,7 & 4,6 & 4,5 \\
4,5 & 4,8 & 4,9 & 4,9 & 4,8 & 4,7 & 4,4 & 4,6 & 4,5 & 4,6 & 4,6 & 4,5 \\
4,5 & 4,7 & 4,8 & 4,8 & 4,7 & 4,6 & 4,4 & 4,5 & 4,4 & 4,6 & 4,5 & 4,4 \\
4,4 & 4,6 & 4,6 & 4,8 & 4,7 & 4,6 & 4,3 & 4,4 & 4,4 & 4,4 & 4,5 & 4,4 \\
4,4 & 4,6 & 4,6 & 4,8 & 4,6 & 4,5 & 4,3 & 4,4 & 4,3 & 4,3 & 4,5 & 4,4 \\
4,3 & 4,5 & 4,4 & 4,7 & 4,5 & 4,5 & 4,3 & 4,4 & 4,3 & 4,3 & 4,4 & 4,4 \\
4,3 & 4,4 & 4,4 & 4,6 & 4,4 & 4,5 & 4,3 & 4,4 & 4,3 & 4,3 & 4,4 & 4,3 \\
4,3 & 4,3 & 4,3 & 4,6 & 4,3 & 4,5 & 4,3 & 4,4 & 4,3 & 4,3 & 4,3 & 4,2 \\
4,3 & 4,3 & 4,2 & 4,5 & 4,3 & 4,4 & 4,2 & 4,4 & 4,3 & 4,2 & 4,2 & 4,2 \\
4,3 & 4,3 & 4,2 & 4,4 & 4,2 & 4,1 & 4,2 & 4,2 & 4,2 & 4,1 & 4,2 & 4,2 \\
4,1 & 4,1 & 4,0 & 4,4 & 4,2 & 4,1 & 4,1 & 4,2 & 4,2 & 4,1 & 4,1 & 4,2 \\
4,0 & 4,1 & 4,0 & 4,3 & 4,2 & 4,1 & 4,1 & 4,2 & 4,2 & 4,0 & 4,1 & 4,1 \\
3,9 & 3,9 & 3,9 & 4,2 & 4,1 & 4,1 & 4,0 & 4,1 & 4,2 & 4,0 & 4,1 & 4,1 \\
3,8 & 3,9 & 3,9 & 4,1 & 4,0 & 4,0 & 3,9 & 4,1 & 4,1 & 4,0 & 4,0 & 4,1 \\
3,7 & 3,8 & 3,9 & 4,1 & 4,0 & 4,0 & 3,8 & 4,1 & 4,1 & 4,0 & 4,0 & 4,1 \\
3,7 & 3,8 & 3,9 & 4,0 & 3,8 & 4,0 & 3,8 & 4,0 & 4,0 & 3,9 & 4,0 & 4,0 \\
3,6 & 3,8 & 3,8 & 3,9 & 3,7 & 3,9 & 3,8 & 3,9 & 4,0 & 3,8 & 4,0 & 4,0 \\
3,5 & 3,8 & 3,8 & 3,8 & 3,7 & 3,9 & 3,8 & 3,9 & 3,9 & 3,8 & 4,0 & 4,0 \\
3,5 & 3,8 & 3,7 & 3,8 & 3,7 & 3,8 & 3,7 & 3,9 & 3,9 & 3,7 & 3,9 & 4,0 \\
3,5 & 3,5 & 3,7 & 3,7 & 3,7 & 3,7 & 3,7 & 3,9 & 3,9 & 3,7 & 3,9 & 3,9 \\
3,5 & 3,5 & 3,7 & 3,6 & 3,4 & 3,7 & 3,7 & 3,8 & 3,8 & 3,7 & 3,9 & 3,8 \\
3,5 & 3,4 & 3,5 & 3,6 & 3,4 & 3,6 & 3,7 & 3,7 & 3,8 & 3,5 & 3,8 & 3,8 \\
3,1 & 3,2 & 3,4 & 3,3 & 3,3 & 3,5 & 3,6 & 3,7 & 3,8 & 3,5 & 3,8 & 3,6 \\
3,1 & 3,2 & 2,8 & 2,9 & 3,1 & 3,3 & 3,3 & 3,3 & 3,7 & 3,5 & 3,8 & 3,5 \\
2,9 & 3,1 & 2,6 & 2,7 & 3,1 & 2,9 & 3,2 & 2,8 & 3,4 & 3,3 & 3,5 & 3,5 \\
2,6 & 2,7 & 2,4 & 2,5 & 2,9 & 2,1 & 2,6 & 2,5 & 2,9 & 2,6 & 3,3 & 3,2 \\
\hline & & & & & & & & & & & \\
\hline
\end{tabular}

*S(5)1- a letra "S" indica que se trata do mês de setembro; o número entre parênteses indica o intervalo de tempo (neste caso 5 dias - pentada); e o último número indica a posição do intervalo de tempo no mês (neste caso, o número 1 indica que se trata do primeiro intervalo). Portanto, $\mathrm{S}(5) 1$ significa: primeira pentada de mês de setembro. 
Tabela 2. Evapotranspiração de referência $(\mathrm{mm} / \mathrm{d})$ obtida através da distribuição Beta para os períodos de 5,10,15 e 30 dias do mês de setembro, em função dos diversos níveis de probabilidade de ocorrência.

\begin{tabular}{ccccccccccccc}
\hline $\mathrm{P}$ & $\mathrm{S}(5)$ & $\mathrm{S}(5)$ & $\mathrm{S}(5)$ & $\mathrm{S}(5)$ & $\mathrm{S}(5)$ & $\mathrm{S}(5)$ & $\mathrm{S}(10$ & $\mathrm{S}(10$ & $\mathrm{S}(10$ & $\mathrm{S}(15$ & $\mathrm{S}(15$ & $\mathrm{S}(3$ \\
\hline $5 \%$ & 2,9 & 3,0 & 2,9 & 2,9 & 3,1 & 2,9 & 3,1 & 3,0 & 3,4 & 3,0 & 3,5 & 3,5 \\
10 & 3,1 & 3,2 & 3,1 & 3,1 & 3,3 & 3,1 & 3,3 & 3,3 & 3,5 & 3,4 & 3,6 & 3,6 \\
20 & 3,4 & 3,5 & 3,4 & 3,5 & 3,5 & 3,5 & 3,6 & 3,6 & 3,8 & 3,6 & 3,8 & 3,8 \\
25 & 3,5 & 3,6 & 3,5 & 3,7 & 3,6 & 3,7 & 3,7 & 3,7 & 3,8 & 3,7 & 3,9 & 3,8 \\
30 & 3,6 & 3,7 & 3,7 & 3,8 & 3,7 & 3,8 & 3,8 & 3,8 & 3,9 & 3,8 & 4,0 & 3,9 \\
40 & 3,8 & 3,9 & 3,9 & 4,1 & 3,9 & 4,0 & 3,9 & 4,0 & 4,0 & 4,0 & 4,1 & 4,0 \\
50 & 4,0 & 4,1 & 4,1 & 4,3 & 4,1 & 4,2 & 4,1 & 4,2 & 4,3 & 4,1 & 4,2 & 4,1 \\
60 & 4,2 & 4,3 & 4,4 & 4,6 & 4,4 & 4,4 & 4,2 & 4,4 & 4,3 & 4,3 & 4,3 & 4,3 \\
70 & 4,4 & 4,6 & 4,6 & 4,8 & 4,6 & 4,6 & 4,4 & 4,5 & 4,4 & 4,4 & 4,4 & 4,4 \\
75 & 4,5 & 4,7 & 4,7 & 4,9 & 4,7 & 4,7 & 4,5 & 4,6 & 4,5 & 4,5 & 4,5 & 4,4 \\
80 & 4,6 & 4,8 & 4,8 & 5,0 & 4,8 & 4,8 & 4,6 & 4,7 & 4,6 & 4,6 & 4,6 & 4,5 \\
90 & 4,9 & 5,1 & 5,1 & 5,1 & 5,1 & 5,1 & 4,8 & 5,0 & 4,9 & 4,7 & 4,7 & 4,6 \\
95 & 5,0 & 5,3 & 5,2 & 5,2 & 5,2 & 5,2 & 5,0 & 5,1 & 5,1 & 4,8 & 4,8 & 4,7 \\
\hline
\end{tabular}

Tabela 3. Estudo comparativo entre a evapotranspiração ao nível de $75 \%$ de probabilidade e a evapotranspiração média, para os períodos de 5,10,15 e 30 dias do mês de setembro (Piracicaba,SP).

\begin{tabular}{ccccc}
\hline Período & $\begin{array}{c}\text { ETo a 75\% } \\
(\mathrm{mm} / \text { dia })\end{array}$ & $\begin{array}{c}\text { ETo média } \\
(\mathrm{mm} / \text { dia })\end{array}$ & $\begin{array}{c}\text { Probabilidade } \\
\text { associada à } \\
\text { ETo média }\end{array}$ & $\begin{array}{c}\text { Relação entre a } \\
\text { ETo a 75\% e a } \\
\text { ETo média }\end{array}$ \\
\hline $\mathrm{S}(5) 1^{*}$ & 4,5 & 4,0 & 50 & 1,125 \\
$\mathrm{~S}(5) 2$ & 4,7 & 4,1 & 50 & 1,146 \\
$\mathrm{~S}(5) 3$ & 4,7 & 4,1 & 50 & 1,146 \\
$\mathrm{~S}(5) 4$ & 4,9 & 4,2 & 45 & 1,167 \\
$\mathrm{~S}(5) 5$ & 4,7 & 4,1 & 50 & 1,146 \\
$\mathrm{~S}(5) 6$ & 4,7 & 4,2 & 50 & 1,119 \\
$\mathrm{~S}(10) 1$ & 4,5 & 4,1 & 50 & 1,098 \\
$\mathrm{~S}(10) 2$ & 4,6 & 4,2 & 50 & 1,095 \\
$\mathrm{~S}(10) 3$ & 4,5 & 4,2 & 50 & 1,071 \\
$\mathrm{~S}(15) 1$ & 4,5 & 4,1 & 50 & 1,098 \\
$\mathrm{~S}(15) 2$ & 4,5 & 4,2 & 50 & 1,071 \\
$\mathrm{~S}(30)$ & 4,4 & 4,1 & 50 & 1,073 \\
\hline
\end{tabular}

O período de máxima exigência hídrica das de forma geral, à medida que diminui o período, 
culturas anuais irrigadas não se restringe a um único dia, ou mesmo, três, cinco ou sete dias. Parece razoável admitir um período variável entre 10 e 30 dias. Jensen (1974) considera que este período, geralmente, compreende duas ou três semanas. Analisando a Tabela 4, verifica-se que, aumenta o valor da evapotranspiração de referência para um mesmo nível de probabilidade, no caso, $75 \%$. A maior diferença entre um dos períodos e o período mensal foi de 11,4\%, justamente um período de 5 dias (as diferenças variaram de 2,3 a 11,4\%).

Tabela 4. Relação entre a evapotranspiração ao nível de $75 \%$ de probabilidade em um determinado período e a evapotranspiração a $75 \%$ de probabilidade para o período de 30 dias, para o mês de setembro (Piracicaba, SP).

\begin{tabular}{ccc}
\hline Periodo & $\begin{array}{c}\text { ETo a } 75 \% \\
(\mathrm{~mm} / \text { dia })\end{array}$ & $\begin{array}{c}\text { \% em relação a ETo a } \\
75 \% \\
\text { do periodo de 30 dias }\end{array}$ \\
\hline $\mathrm{S}(5) 1$ & 4,5 & 2,3 \\
$\mathrm{~S}(5) 2$ & 4,7 & 6,8 \\
$\mathrm{~S}(5) 3$ & 4,7 & 6,8 \\
$\mathrm{~S}(5) 4$ & 4,9 & 11,4 \\
$\mathrm{~S}(5) 5$ & 4,7 & 6,8 \\
$\mathrm{~S}(5) 6$ & 4,7 & 6,8 \\
$\mathrm{~S}(10) 1$ & 4,5 & 2,3 \\
$\mathrm{~S}(10) 2$ & 4,6 & 4,5 \\
$\mathrm{~S}(10) 3$ & 4,5 & 2,3 \\
$\mathrm{~S}(15) 1$ & 4,5 & 2,3 \\
$\mathrm{~S}(15) 2$ & 4,5 & 2,3 \\
$\mathrm{~S}(30)$ & 4,4 & 0,0 \\
\hline
\end{tabular}

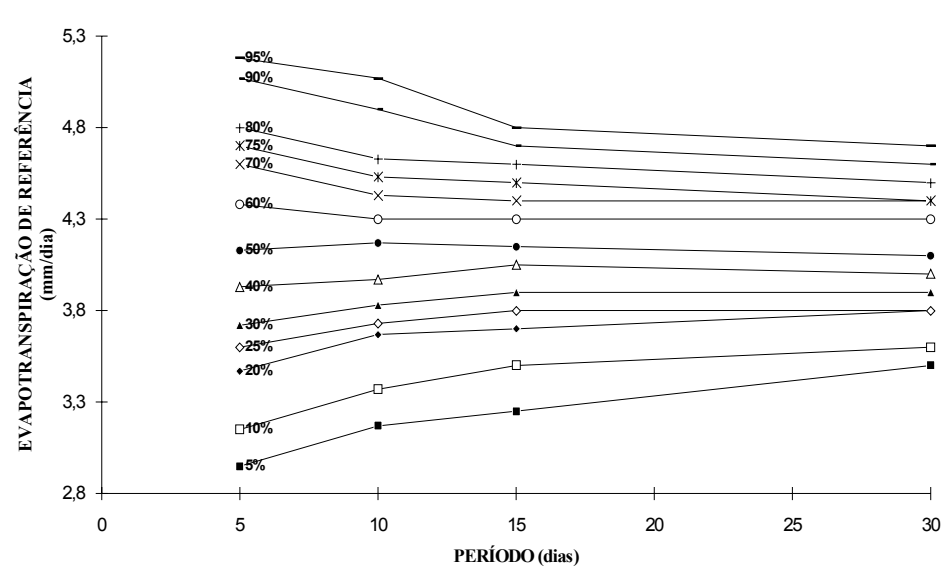

Figura 1. Evapotranspiração de referência para os diferentes níveis de probabilidade de ocorrência, em função da duração do período.

Pela Figura 1, verifica-se que o aumento do valor da evapotranspiração de referência com a diminuição do período ocorre para valores de de Agricultura Luiz de Queiroz, USP, 1983. $349 \mathrm{p}$.

DOORENBOS, J., PRUITT, W.O. Guidelines for 
probabilidade maiores ou iguais a $60 \%$. Portanto, a prática de se adotar o máximo valor diário da evapotranspiração no período de pico acarreta o superdimensionamento dos sistemas de irrigação, quando comparado com o valor recomendado, correspondente ao nível de $75 \%$ de probabilidade.

\section{CONCLUSÕES}

As distribuições Beta e Normal foram aptas para representar os dados de evapotranspiração de referência do mês de setembro, relativos a Piracicaba, SP.

Para valores de probabilidade de ocorrência iguais ou superiores a $60 \%$, verificou-se o aumento do valor da evapotranspiração de referência com a diminuição do período.

Considerando que o critério recomendado é aquele que estabelece o período de pico em termos de exigência hídrica como sendo de 2 a 3 semanas e que adota a evapotranspiração de referência ao nível de $75 \%$ de probabilidade de ocorrência, temse que a prática usual de se utilizar o valor médio mensal da evapotranspiração no dimensionamento de sistemas de irrigação conduz ao subdimensionamento dos mesmos, enquanto a adoção do máximo valor diário da evapotranspiração no período de pico acarreta o superdimensionamento.

\section{REFERÊNCIAS BIBLIOGRÁFICAS}

ARRUDA, F.B., BARROSO, L.F.S. Estimativa do uso de água para fins de projeto de irrigação, em função da evaporação de tanque, em Ribeirão Preto. Bragantia, v.43, p.677-82, 1984.

CAMPOS, H. Estatística experimental nãoparamétrica. 4.ed. Piracicaba: Escola Superior predicting crop water requirements. Rome:FAO, 1984. 144p. (Irrigation and Drainage Paper, 24).

FALLS, L.W. The Beta distribution: a statistical model for world cloud cover. Alabama: NASA, 1973. 6p. (NASA Technical Memorandum, TMX-64714).

JENSEN, M.E. Consumptive use of water and irrigation water requirements. New York: ASCE, 1974. 215p.

NIXON, P.R., LAWLESS, G.P., RICHARDSON, G.V. Coastal California evapotranspiration frequences. Drainage Division, v.98, n.IR2, p.182-91, 1972.

PEARSON, K. Tables of the incomplete Betafunction. London: University College, Biometrika Office, 1934. 494p.

PRUITT, W.O., OETTINGEN, S., MORGAN, D.L. Central California evapotranspiration frequences. Drainage Division, v.98, n.IR2, p.177-84, 1972.

SAAD, J.C.C. Estudo das distribuições de freqüencia da evapotranspiração de referência e da precipitação pluvial para fins de dimensionamento de sistemas de irrigação. Piracicaba, 1990. 124p. Dissertação (Mestrado em Irrigação e Drenagem) - Escola Superior de Agricultura "Luiz de Queiroz", Universidade de São Paulo.

SAAD, J.C.C. , SCALOPPI, E.J. Freqüência de distribuição de evapotranspiração de referência para dimensionamento de sistemas de irrigação. In: CONGRESSO NACIONAL DE IRRIGAÇÃO E DRENAGEM, 8, 1988, Florianópolis. Anais... Florianópolis: Associação Brasileira de Irrigação e Drenagem, 1988. v.2, p.1037-52.

SPIEGEL, M.R. Estatística. 2.ed. São Paulo: McGraw-Hill do Brasil, 1985. 453p.

WRIGHT, J.L., JENSEN, M.E. Peak water requirements in Southern Idaho. Drainage Division, v.98, n.IR2, p.193-201, 1972.

YAO, A.Y.M. The R index for plant water requirement. Agricultural Meteorology, Amsterdam, n.6, p.259-73, 1969. 